\title{
Outcomes after left ventricular assist device implantation in patients with acute kidney injury
}

\author{
Samuel A. Silver, MD, MSc, , ${ }^{\text {,b }}$ Jin Long, PhD, ${ }^{\mathrm{b}}$ Yuanchao Zheng, MS, ${ }^{\mathrm{b}}$ Andrew B. Goldstone, MD, PhD, \\ Doug Franz, MD, MPH, ${ }^{\mathrm{b}}$ Tara I. Chang, MD, MS, ${ }^{\mathrm{b}}$ and Glenn M. Chertow, MD, MPH ${ }^{\mathrm{b}}$
}

\section{ABSTRACT}

Objective: The study objective was to compare outcomes for patients with and without acute kidney injury during hospitalizations when left ventricular assist devices are implanted.

Methods: By using the National Inpatient Sample from 2008 to 2013, we identified patients with an International Classification of Diseases, Ninth Revision procedure code for left ventricular assist device implantation (37.66). We ascertained the presence of acute kidney injury and acute kidney injury requiring dialysis using validated International Classification of Diseases, Ninth Revision codes. We used logistic regression to examine the association of nondialysis-requiring acute kidney injury and acute kidney injury requiring dialysis with mortality, procedural complications, and discharge destination.

Results: We identified 8362 patients who underwent left ventricular assist device implantation, of whom $3760(45.0 \%)$ experienced nondialysis-requiring acute kidney injury and $426(5.1 \%)$ experienced acute kidney injury requiring dialysis. In-hospital mortality was $3.9 \%$ for patients without acute kidney injury, $12.2 \%$ for patients with nondialysis-requiring acute kidney injury, and $47.4 \%$ for patients with acute kidney injury requiring dialysis. Patients with nondialysisrequiring acute kidney injury and acute kidney injury requiring dialysis had higher adjusted odds of mortality $(3.24,95 \%$ confidence interval [CI], 2.04-5.13 and 20.8, 95\% CI, 9.7-44.2), major bleeding (1.38, 95\% CI, 1.08-1.77 and 2.44, $95 \%$ CI, 1.47-4.04), sepsis $(2.69,95 \%$ CI, $1.93-3.75$ and 5.75, 95\% CI, $3.46-9.56)$, and discharge to a nursing facility $(2.15,95 \% \mathrm{CI}, 1.51-3.07$ and 5.89, 95\% CI, 2.67-12.99).

Conclusions: More than 1 in 10 patients with acute kidney injury and approximately 1 in 2 patients with acute kidney injury requiring dialysis died during their hospitalization, with only $30 \%$ of patients with acute kidney injury requiring dialysis discharged to home. This information is necessary to support shared decision-making for patients with advanced heart failure and acute kidney injury. (J Thorac Cardiovasc Surg 2020;159:477-86)

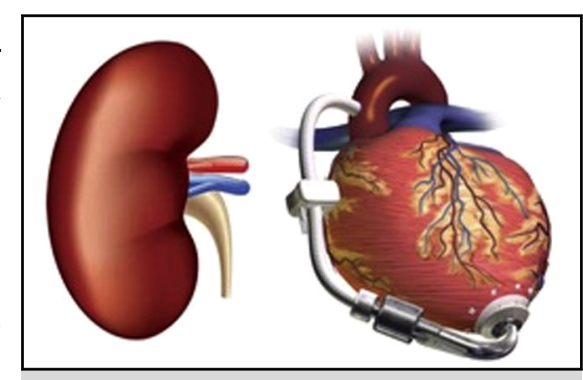

LVADs in patients with AKI.

\section{Central Message}

One in 10 patients with AKI and 1 in 2 patients with AKI-D will die in hospital after LVAD implantation. This informs shared decisionmaking, particularly for patients unable to stop dialysis.

\section{Perspective}

In this nationally representative study of patients who underwent LVAD implantation from 2008 to 2013, we found that half of patients experienced AKI and that it was a major risk factor for in-hospital mortality. Additional studies are needed to stratify outcomes by LVAD indication and the timing of AKI onset. Our data should be used to improve decisionaids for patients considering LVAD implantation.

See Commentaries on pages 487 and 489.
Left ventricular assist devices (LVADs) are increasingly deployed for patients with advanced heart failure as a bridge to heart transplantation or destination therapy because of their ability to prolong survival and enhance quality of life. ${ }^{1,2}$ In the United States, the annual LVAD implantation rate exceeds 2000 per year and since 2009 has surpassed the

\footnotetext{
From the a'Division of Nephrology, Kingston Health Sciences Center, Queen's University, Kingston, Ontario, Canada; ' ${ }^{\mathrm{b}}$ Division of Nephrology, Stanford University School of Medicine, Stanford, Calif; and ${ }^{\mathrm{c}}$ Department of Cardiothoracic Surgery, Stanford University School of Medicine, Stanford, Calif.

S.A.S. was supported by a Kidney Research Scientist Core Education and National Training Program Post-Doctoral Fellowship (co-funded by the Kidney Foundation of Canada, Canadian Society of Nephrology, and Canadian Institutes of Health Research). G.M.C. was supported by a K24 mid-career mentoring award from National Institute of Diabetes and Digestive and Kidney Diseases (K24 DK085446). These funders had no role in the design and conduct of the study;
} 


\begin{tabular}{|c|c|}
\hline \multicolumn{2}{|c|}{ Abbreviations and Acronyms } \\
\hline AKI & $=$ acute kidney injury \\
\hline AKI-D & $=$ acute kidney injury requiring dialysis \\
\hline AKI-NL & $\begin{array}{l}=\text { nondialysis-requiring acute kidney } \\
\text { injury }\end{array}$ \\
\hline $\mathrm{CI}$ & $=$ confidence interval \\
\hline CKD & $=$ chronic kidney disease \\
\hline ESRD & $=$ end-stage renal disease \\
\hline ICD-9 & $\begin{aligned}= & \text { International Classification of Diseases, } \\
& \text { Ninth Revision }\end{aligned}$ \\
\hline LVAD & $=$ left ventricular assist device \\
\hline NIS & $=$ National Inpatient Sample \\
\hline OR & $=$ odds ratio \\
\hline PE & $=$ pulmonary embolus \\
\hline
\end{tabular}

(U) Scanning this QR code will
take you to the article title
page to access supplementary
information.

number of heart transplants. ${ }^{3,4}$ Due in part to technologic advances and a shortage of donor hearts, these and related devices are being implanted in patients with multiple comorbidities by a growing number of centers. ${ }^{1,3,5}$

The benefits of LVAD implantation must be balanced against the risks of device-related complications. Acute kidney injury (AKI) is one of the more common complications, with an overall incidence that ranges from $7 \%$ to $56 \%$ and an incidence of AKI requiring dialysis (AKI-D) as high as $33 \%{ }^{6,7}$ AKI after LVAD implantation is also associated with a more complicated hospital course, including an increased risk of major bleeding and stroke ${ }^{7}$ and 3-month mortality rates that may approach $50 \%{ }^{8}$

Despite these poor outcomes, there are few multicenter studies that have examined the incidence of AKI and its association with adverse events after LVAD implantation. Nationally representative data are required to determine the burden of AKI at a time when LVADs are being implanted in more patients for increasing indications, sometimes by less-experienced providers. ${ }^{3,5,9}$ To address this knowledge gap, we used the National Inpatient Sample (NIS) to better understand practice patterns across the United States for patients with LVADs and AKI. Our objectives were to (1) determine the incidence rate of AKI during a hospitalization in which an LVAD is implanted, (2) identify risk factors for AKI, and (3) compare in-hospital outcomes for patients with AKI relative to patients without AKI. We hypothesized that patients with AKI and particularly AKI-D would be at increased risk of procedural complications and death, even after accounting for other risk factors, comorbid conditions, and disease severity.

\section{MATERIALS AND METHODS \\ Study Population}

We extracted data from the NIS, a nationally representative administrative database of hospitalizations in the United States created by the Agency for Healthcare Research and Quality as part of the Healthcare Cost and Utilization Project. ${ }^{10}$ The NIS is the largest all-payer inpatient database in the United States. Data are stratified according to geographic region, location (urban/rural), teaching status, ownership, and hospital bed number. Each hospitalization is treated as an individual entry in the database, such that individual patients who are hospitalized multiple times may be present in the NIS multiple times. Sample weights are provided to allow for the generation of national estimates, along with information necessary to calculate the variance of estimates.

We used the 2008 to 2013 NIS subsets, with 2013 the most recent year available at the time of data analysis. This timeframe coincided with the approval of LVADs for destination therapy and the predominant use of continuous-flow devices. ${ }^{11}$ The 2008 to 2013 NIS subsets contained administrative data from more than 42 million hospitalizations, representing more than 40 states and $95 \%$ of the US population. ${ }^{10}$

We included all patients aged 18 years or more with an International Classification of Diseases, Ninth Revision (ICD-9) procedure code for implantation of an LVAD (37.66). We excluded patients with ICD-9 codes for end-stage renal disease (ESRD), as well as patients with an ICD-9 code for dialysis but without a diagnosis code for AKI, assuming that these patients received dialysis for ESRD. ${ }^{12-14}$ To ensure that we captured isolated LVAD implantation, we also excluded patients with evidence of cardiac surgery procedures, biventricular assist devices, and short-term mechanical circulatory support placement during the same hospitalization as LVAD implantation (Table E1).

\section{Primary Exposure}

We identified episodes of AKI using ICD-9 code 584.x (Table E1). This administrative code for AKI has a specificity of approximately $99 \%$ and identifies a more severe spectrum of AKI compared with AKI defined using serum creatinine criteria. ${ }^{14,15}$ For example, the median (25th, 75th percentile) change in serum creatinine from baseline is estimated at 1.2 $(0.7-2.1) \mathrm{mg} / \mathrm{dL}$ compared with $0.2(0.1-0.2) \mathrm{mg} / \mathrm{dL}$ for patients without an administrative code for AKI. ${ }^{14}$ We defined AKI-D as the presence of an AKI diagnosis code and a diagnosis or procedure code for dialysis; we defined patients without a dialysis code as nondialysis-requiring AKI (AKI-ND). This algorithm for AKI-D has been shown to yield high sensitivity and specificity. ${ }^{14}$

\section{Outcomes}

The primary outcome was in-hospital death. Secondary outcomes included procedural complications (major bleeding, postoperative sepsis, postoperative stroke, postoperative deep vein thrombosis or pulmonary embolus [PE], systemic embolism, respiratory complications, and cardiac complications), discharge disposition, and length of stay. We defined procedural complications using validated Patient Safety Indicators from the Agency for Healthcare Research and Quality or ICD-9 codes from other LVAD studies (Table E2). ${ }^{1,5}$ Heart transplantation was ascertained using ICD-9 procedure code 37.51 .

\section{Covariates}

Demographic variables included patient age, sex, race, health insurance status, and median household income by ZIP code. Hospital-level variables included geographic region, bed number, teaching status, and hospital ownership using predetermined NIS definitions. ${ }^{10}$ We used ICD-9 
diagnostic and procedure codes to identify the following comorbid conditions: atrial fibrillation, cancer, chronic kidney disease (CKD), chronic obstructive pulmonary disease, heart failure, coronary artery disease, dementia, diabetes mellitus, history of coronary artery bypass grafting, history of percutaneous coronary intervention, hypertension, liver disease, peripheral vascular disease, and valvular heart disease. We used a similar approach to ascertain the following details from the acute hospitalization: acute respiratory distress syndrome, cardiogenic shock, cardioplegia, closure of septal defects, intra-aortic balloon pump, mechanical ventilation, and Swan-Ganz catheter insertion (Table E1).

\section{Statistical Analysis}

We summarized baseline characteristics of the study participants using descriptive statistics. We expressed normally distributed continuous variables as mean (standard deviation) and non-normally distributed continuous variables as median (25th, 75th percentile range). We expressed categoric variables as proportions.

We used logistic regression to evaluate associations among different covariates and AKI. These models included the demographic information, hospital-level variables, patient comorbidities, and hospitalization details listed in Table 1. We used single models for each variable to estimate the unadjusted odds ratio (OR) and $95 \%$ confidence interval $(\mathrm{CI})$. We used backwards stepwise selection to identify variables jointly associated with AKI-ND and AKI-D. As a form of case-mix adjustment, we accounted for risk factors associated with AKI when we determined the number of LVADs implanted in patients each year. Next, we used logistic regression models to examine the association of AKI-ND and AKI-D (vs no AKI) with the primary and secondary outcomes, adjusting for the covariates in Table 1. To further estimate the effect of AKI on mortality, we adjusted the models for all procedural complications.

All analyses presented account for the NIS complex survey design (via weighting, stratification, and clustering), subpopulation measurements to generate national estimates, and changes in the data structure over time. We created the cohort using SAS software version 9.4 (SAS Institute, Inc, Cary, NC) and conducted the analyses using StataMP version 14.0 (StataCorp LP, College Station, Tex).

\section{RESULTS}

\section{Patient Characteristics}

Between January 1, 2008, and December 31, 2013, there were 15,150 hospitalization records available in the NIS for patients aged 18 years or more who underwent LVAD implantation; 420 records $(2.8 \%)$ were classified as having ESRD, 6368 records $(42.0 \%)$ underwent concomitant cardiac surgery, and 8362 records $(55.2 \%)$ were included in the final cohort. Within the final cohort, 4186 (50.1\%) hospitalizations were complicated by AKI, of which 426 $(10.2 \%)$ required dialysis (corresponding to $5.1 \%$ of the analytic cohort) (Figure 1).

The number of LVADs implanted increased from 786 in 2008 to 1975 in 2013 (Figure 2). The proportion of patients with AKI-ND increased from $43.5 \%$ in 2008 to $48.6 \%$ in 2013, with little change in the proportion of patients with AKI-D (5.1\% and $4.6 \%$ in the corresponding years). These numbers remained essentially unchanged after adjustment for age, sex, and factors associated with AKI (Table E3).

Patients with AKI-ND and AKI-D had a mean age of 56.8 (0.5) and 54.8 (1.4) years, with $82.4 \%$ and $80.7 \%$ of patients of male sex. Patients without AKI had a mean age of $55.6(0.5)$ years, with $76.6 \%$ of male sex. More than $90 \%$ of all LVADs were implanted in patients in urban teaching hospitals with large bed numbers. Compared with patients without AKI, patients with AKI-ND and AKI-D were more like to be black $(24.0 \%$ and $24.8 \%$ vs $20.3 \%)$ and have CKD (51.1\% and $53.4 \%$ vs $30.4 \%)$; they were less likely to have valvular heart disease (22.2\% and $13.8 \%$ vs $30.6 \%$ ).

The index hospitalization in patients with AKI-ND and AKI-D (vs patients without AKI) was also more likely to involve a nonelective admission $(71.0 \%$ and $69.9 \%$ vs $57.4 \%)$, acute respiratory distress syndrome $(27.1 \%$ and $61.9 \%$ vs $16.4 \%$ ), cardiogenic shock $(53.9 \%$ and $62.1 \%$ vs $36.6 \%$ ), an intra-aortic balloon pump $(26.7 \%$ and $30.8 \%$ vs $17.6 \%)$, mechanical ventilation (34.0\% and $67.6 \%$ vs $23.6 \%$ ), and Swan-Ganz insertion (34.9\% and $36.6 \%$ vs $28.1 \%$ ) (Table 1). Accordingly, the median time from hospital admission to LVAD implantation was longer in patients with AKI-ND (8 [4-15] days) and AKI-D (7 [2-12] days) than in patients without AKI (5 [2-10] days). Longer implantation times were also observed in elective and nonelective hospitalizations for patients with AKI-ND (5 [2-11] days and 10 [5-16] days) and AKI-D (2 [1-8] days and 9 [4-17] days) vs patients without AKI (2 [1-7] days and 7 [4-14] days).

\section{Determinants of Acute Kidney Injury}

Table 2 shows factors associated with AKI-ND and AKI$\mathrm{D}$ in the multivariable model. Older age was associated with a higher risk of AKI-ND (OR, 1.09 per decade; 95\% CI, 1.01-1.16) but not AKI-D (OR, 0.90 per decade; $95 \%$ CI, 0.74-1.10). The comorbid condition most strongly associated with AKI was CKD, with a 3-fold higher risk of AKI-ND (OR, 3.17; 95\% CI, 2.50-4.02) and 4-fold higher risk of AKI-D (OR, 4.23; 95\% CI, 2.69-6.64). The presence of hypertension or valvular heart disease was associated with a lower risk of AKI-ND (OR, 0.60; 95\% CI, 0.45 0.78 and OR, 0.67; 95\% CI, 0.52-0.87) and AKI-D (OR, 0.57 ; $95 \%$ CI, $0.35-0.93$ and OR, 0.43 ; $95 \%$ CI, $0.22-$ 0.83 ), respectively. Relative to a nonelective hospital admission, an elective hospital admission was associated with a lower risk of AKI-ND (OR, 0.58; 95\% CI, 0.470.72 ) but not AKI-D (OR, 0.66; 95\% CI, 0.41-1.07). Cardiogenic shock and mechanical ventilation were the only in-hospital events associated with a higher risk of AKI-ND (OR, 1.81; 95\% CI, 1.42-2.30 and OR, 1.52; 95\% CI, 1.21-1.91) and AKI-D (OR, 2.02; 95\% CI, 1.183.44 and $\mathrm{OR}, 6.11 ; 95 \% \mathrm{CI}, 3.74-10.00)$.

\section{Patient Outcomes}

In-hospital mortality was 3.9\% for patients without AKI, $12.2 \%$ for patients with AKI-ND, and $47.4 \%$ for patients with AKI-D (Table 3). We observed similar increases for most procedural complications, including major bleeding, 
TABLE 1. Baseline characteristics of the cohort

\begin{tabular}{|c|c|c|c|}
\hline Characteristics & No $A K I, N=4176$ & AKI-ND, N = 3760 & AKI-D, N $=426$ \\
\hline \multicolumn{4}{|l|}{ Demographics } \\
\hline Age (mean, SD), in years & $55.6 \pm 0.5$ & $56.8 \pm 0.5$ & $54.8 \pm 1.4$ \\
\hline \multicolumn{4}{|l|}{ Sex, $\%$} \\
\hline Male & 76.6 & 82.4 & 80.7 \\
\hline Female & 23.4 & 17.6 & 19.3 \\
\hline \multicolumn{4}{|l|}{ Race, $\%$} \\
\hline White & 68.6 & 62.6 & 56.0 \\
\hline Black & 20.3 & 24.0 & 24.8 \\
\hline Hispanic & 5.8 & 5.9 & 6.1 \\
\hline Asian or Pacific Islander & 1.8 & 1.8 & 3.7 \\
\hline Native American & 0.1 & 0.3 & 0.0 \\
\hline Other & 3.4 & 5.4 & 9.4 \\
\hline \multicolumn{4}{|l|}{ Median household income for patient's ZIP code, $\%$} \\
\hline$<\$ 39,000$ & 25.8 & 23.9 & 29.7 \\
\hline$\$ 39,000-\$ 47,999$ & 23.4 & 28.3 & 21.2 \\
\hline$\$ 48,000-\$ 62,999$ & 27.5 & 26.9 & 24.6 \\
\hline$\geq \$ 63,000$ & 23.3 & 20.9 & 24.5 \\
\hline \multicolumn{4}{|l|}{ Hospital-level variables, $\%$} \\
\hline \multicolumn{4}{|l|}{ Hospital region } \\
\hline Northeast & 21.2 & 15.7 & 10.4 \\
\hline Midwest & 27.3 & 28.5 & 35.2 \\
\hline South & 37.8 & 40.6 & 32.2 \\
\hline West & 13.7 & 15.2 & 22.2 \\
\hline \multicolumn{4}{|l|}{ Hospital bed number } \\
\hline Small & 1.3 & 0.5 & 4.6 \\
\hline Medium & 7.8 & 8.8 & 10.6 \\
\hline Large & 90.9 & 90.7 & 84.8 \\
\hline \multicolumn{4}{|l|}{ Hospital teaching status } \\
\hline Rural & 0.1 & 0.0 & 0.0 \\
\hline Urban nonteaching & 2.8 & 1.4 & 5.6 \\
\hline Urban teaching & 97.1 & 98.6 & 94.4 \\
\hline \multicolumn{4}{|l|}{ Ownership } \\
\hline Government nonfederal & 13.8 & 17.7 & 17.2 \\
\hline Private nonprofit & 83.5 & 78.8 & 78.2 \\
\hline Private investor-owned & 2.7 & 3.5 & 4.6 \\
\hline \multicolumn{4}{|l|}{ Comorbidities, $\%$} \\
\hline Atrial fibrillation & 39.3 & 45.0 & 40.3 \\
\hline Cancer & 3.1 & 3.0 & 2.4 \\
\hline CKD & 30.4 & 51.1 & 53.4 \\
\hline Chronic obstructive pulmonary disease & 13.3 & 12.5 & 3.5 \\
\hline Congestive heart failure & 96.3 & 97.7 & 94.3 \\
\hline Coronary artery disease & 56.6 & 52.8 & 54.2 \\
\hline Dementia & 0.1 & 0.1 & 0.0 \\
\hline Diabetes & 32.8 & 30.0 & 34.5 \\
\hline History of coronary artery bypass grafting & 9.9 & 8.9 & 3.5 \\
\hline History of percutaneous coronary intervention & 2.5 & 2.5 & 3.2 \\
\hline Hypertension & 51.3 & 48.6 & 47.9 \\
\hline Liver disease & 11.4 & 14.4 & 20.8 \\
\hline Peripheral vascular disease & 4.6 & 4.9 & 1.2 \\
\hline Valvular heart disease & 30.6 & 22.2 & 13.8 \\
\hline \multicolumn{4}{|l|}{ Details of acute hospitalization, $\%$} \\
\hline \multicolumn{4}{|l|}{ Admission type } \\
\hline Nonelective & 57.4 & 71.0 & 69.9 \\
\hline Elective & 42.6 & 29.0 & 30.1 \\
\hline
\end{tabular}


TABLE 1. Continued

\begin{tabular}{lccc}
\hline \multicolumn{1}{c}{ Characteristics } & No AKI, N $=\mathbf{4 1 7 6}$ & AKI-ND, N $=\mathbf{3 7 6 0}$ & AKI-D, N $=\mathbf{4 2 6}$ \\
\hline Acute respiratory distress syndrome & 16.4 & 27.1 & 61.9 \\
Cardiogenic shock & 36.6 & 53.9 & 62.1 \\
Cardioplegia & 1.0 & 0.5 & 0.0 \\
Closure of septal defects & 4.3 & 4.3 & 4.7 \\
Intra-aortic balloon pump & 17.6 & 26.7 & 30.8 \\
Mechanical ventilation & 23.6 & 34.0 & 67.6 \\
Swan-Ganz insertion & 28.1 & 34.9 & 36.6 \\
\hline
\end{tabular}

$A K I$, Acute kidney injury; $A K I-N D$, nondialysis-requiring acute kidney injury; $A K I-D$, acute kidney injury-requiring dialysis; $S D$, standard deviation; $C K D$, chronic kidney disease.

postoperative sepsis, postoperative deep vein thrombosis/ PE, systemic embolism, and cardiac complications. Among patients who survived to hospital discharge, the median length of stay and hospital costs were longer for patients with AKI-ND (29 [21-45] days; \$191,225 [\$152,266\$244,190]) and AKI-D (44 [33-53] days; \$270,579 [\$222,790-\$326,201]) than patients without AKI (23 [1533] days; $\$ 164,760$ [\$133,728-\$202,765]). Patients with AKI-ND (72.3\%) and AKI-D (56.3\%) were also less likely to be discharged home than patients without AKI (83.6\%). None of the patients with AKI-D received a heart transplant during the same hospitalization as LVAD implantation.

After multivariable adjustment (Figure 3), patients with AKI-ND and AKI-D had higher odds of mortality (OR, 3.24; 95\% CI, 2.04-5.13 and OR, 20.8; 95\% CI, 9.744.2), major bleeding (OR, 1.38; 95\% CI, 1.08-1.77 and OR, 2.44; 95\% CI, 1.47-4.04), postoperative sepsis (OR,
2.69; 95\% CI, 1.93-3.75 and OR, 5.75; 95\% CI, 3.469.56), and discharge to a nursing facility (OR, 2.15; $95 \%$ CI, 1.51-3.07 and OR, 5.89; 95\% CI, 2.67-12.99). The strong association between AKI and death was maintained even after adjustment for procedural complications (AKIND, OR, 2.41; 95\% CI, 1.50-3.88 and AKI-D, OR, 15.5; 95\% CI, 7.1-34.1). We also observed similar results when the analysis was restricted to the 302 patients with AKI-D in whom we could ascertain the timing of LVAD implantation (75 with pre-LVAD AKI-D and 227 with post-LVAD AKI-D); respective ORs for death were 21.2 (95\% CI, 6.0-74.2) for preoperative AKI-D and 29.7 (95\% CI, 12.4-70.9) for postoperative AKI-D.

In a post hoc analysis of patients who received mechanical ventilation, outcomes were extremely poor. The mortality rate was $32 \%$ and $70 \%$ in patients with AKI-ND and AKI-D, respectively. After multivariable adjustment,

- 15,150 patient records in NIS from Jan 1, 2008 to Dec 31, 2013 for patients $\geq 18$ years of age with ICD-9 code 37.66 for implantation of LVAD

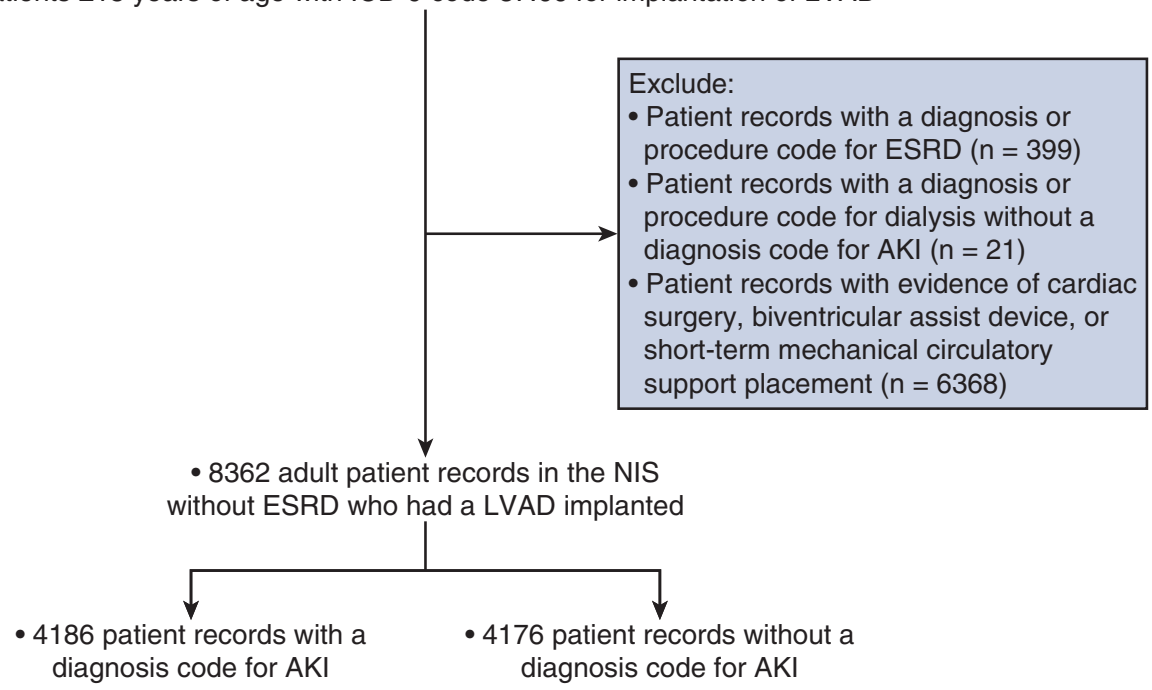

FIGURE 1. Inclusion and exclusion criteria used to define a cohort of adult patients without ESRD who had an LVAD implanted. To ensure that we captured isolated LVAD implantation, we excluded patients with evidence of cardiac surgery procedures, biventricular assist devices, and short-term mechanical circulatory support placement during the same hospitalization as LVAD implantation. After exclusions, we identified 8362 patients who underwent LVAD implantation, of whom 4186 (50.1\%) experienced AKI. NIS, National Inpatient Sample; ICD-9, International Classification of Diseases, Ninth Revision; LVAD, left ventricular assist device; ESRD, end-stage renal disease; $A K I$, acute kidney injury. 


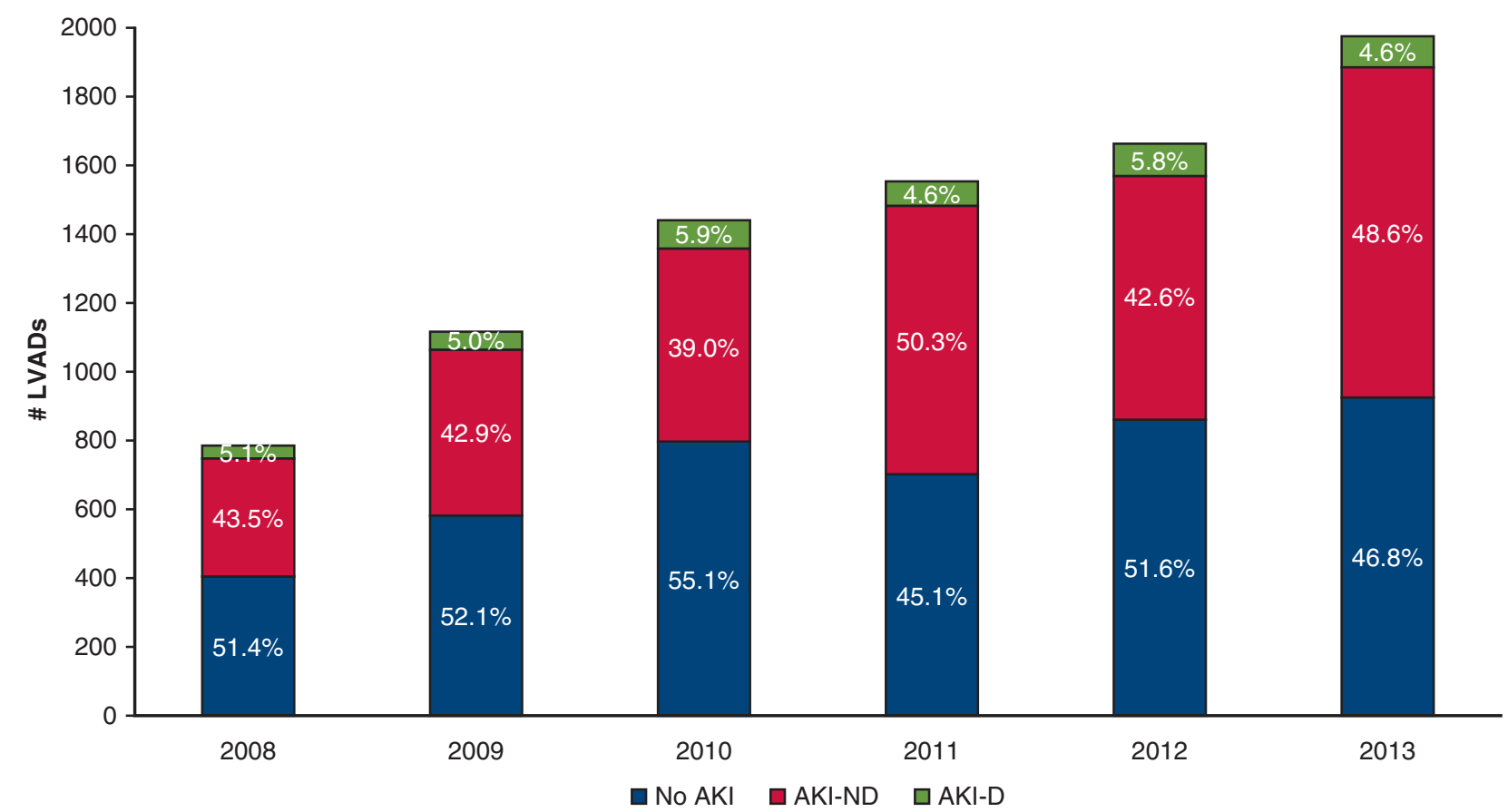

FIGURE 2. Number of LVADs implanted stratified by year and AKI. The colored bars represent the percentage of patients with and without AKI. The number of LVADs implanted increased from 786 in 2008 to 1975 in 2013. The proportion of patients with AKI-ND increased from $43.5 \%$ in 2008 to $48.6 \%$ in 2013, with little change in the proportion of patients with AKI-D (5.1\% and 4.6\% in 2008 and 2013, respectively). LVAD, Left ventricular assist device; $A K I$, acute kidney injury; $A K I-D$, AKI-requiring dialysis; $A K I-N D$, nondialysis-requiring AKI.

patients with AKI-ND and AKI-D had a 14-fold (OR, 14.2; 95\% CI, 7.5-26.9) and 90-fold (OR, 90.9; 95\% CI, 41.4199.6) higher risk of death if on mechanical ventilation.

\section{DISCUSSION}

Among a nationally representative cohort of patients who underwent LVAD implantation from 2008 to 2013, more than 1 in 10 patients with AKI-ND and approximately 1 in 2 patients with AKI-D died during their hospital stay. AKI was associated with higher odds of major bleeding, postoperative sepsis, and discharge to a nursing facility, with only $30 \%$ of patients with AKI-D able to return directly home. Moreover, we observed that the number of LVADs implanted increased each year while the rate of AKI remained stable at approximately $50 \%$. These findings suggest that AKI is not decreasing despite improved LVAD technology, which reinforces the need to identify predictors of AKI and adverse outcomes.

Several single-center studies have examined the relations among pre-LVAD kidney function, AKI, and outcomes. ${ }^{6,16}$

TABLE 2. Determinants of acute kidney injury among patients who underwent implantation of a left ventricular assist device

\begin{tabular}{|c|c|c|c|c|}
\hline & \multicolumn{2}{|c|}{ AKI-ND } & \multicolumn{2}{|c|}{ AKI-D } \\
\hline & $\begin{array}{c}\text { Unadjusted } \\
(\mathrm{OR}, 95 \% \mathrm{CI})\end{array}$ & $\begin{array}{c}\text { Adjusted } \\
(\mathrm{OR}, \mathbf{9 5} \% \mathrm{CI})\end{array}$ & $\begin{array}{c}\text { Unadjusted } \\
(\mathrm{OR}, 95 \% \mathrm{CI})\end{array}$ & $\begin{array}{c}\text { Adjusted } \\
(\mathrm{OR}, \mathbf{9 5} \% \mathrm{CI})\end{array}$ \\
\hline \multicolumn{5}{|l|}{ Demographics } \\
\hline Age (per $10 \mathrm{y})$ & $1.10(1.00-1.15)$ & $1.09(1.01-1.16)$ & $1.00(0.82-1.10)$ & $0.90(0.74-1.10)$ \\
\hline \multicolumn{5}{|l|}{ Comorbidities } \\
\hline CKD & $2.40(1.93-2.98)$ & $3.17(2.50-4.02)$ & $2.62(1.62-4.23)$ & $4.23(2.69-6.64)$ \\
\hline Hypertension & $0.90(0.70-1.15)$ & $0.60(0.45-0.78)$ & $0.87(0.52-1.46)$ & $0.57(0.35-0.93)$ \\
\hline Valvular heart disease & $0.65(0.51-0.82)$ & $0.67(0.52-0.87)$ & $0.36(0.19-0.69)$ & $0.43(0.22-0.83)$ \\
\hline \multicolumn{5}{|l|}{ Details of acute hospitalization } \\
\hline Elective admission (vs nonelective) & $0.55(0.44-0.69)$ & $0.58(0.47-0.72)$ & $0.58(0.36-0.92)$ & $0.66(0.41-1.07)$ \\
\hline Cardiogenic shock & $2.03(1.62-2.54)$ & $1.81(1.42-2.30)$ & $2.83(1.69-4.76)$ & $2.02(1.18-3.44)$ \\
\hline Mechanical ventilation & $1.67(1.34-2.08)$ & $1.52(1.21-1.91)$ & $6.76(4.25-10.75)$ & $6.11(3.74-10.00)$ \\
\hline
\end{tabular}

All estimates are adjusted for the demographic factors, hospital-level variables, comorbidities, and hospitalization details listed in Table 1. AKI-ND, Nondialysis-requiring acute kidney injury; $A K I-D$, acute kidney injury-requiring dialysis; $O R$, odds ratio; $C I$, confidence interval; $C K D$, chronic kidney disease. 
TABLE 3. Patient outcomes

\begin{tabular}{lccc}
\hline \multicolumn{1}{c}{ Outcome } & No AKI, $\mathbf{N}=\mathbf{4 1 7 6}$ & AKI-ND, N $=\mathbf{3 7 6 0}$ & AKI-D, N=426 \\
\hline In-hospital mortality, (n, \%) & $162(3.9)$ & $457(12.2)$ & $202(47.4)$ \\
Procedural complications, (n, \%) & & & \\
$\quad$ Major bleeding & $1058(25.3)$ & $1233(32.8)$ & $211(49.5)$ \\
$\quad$ Postoperative sepsis & $357(8.5)$ & $843(22.4)$ & $181(42.5)$ \\
$\quad$ Postoperative stroke & $229(5.5)$ & $195(5.2)$ & $25(5.9)$ \\
$\quad$ Postoperative DVT/PE & $344(8.2)$ & $395(10.5)$ & $73(17.1)$ \\
Systemic embolism & $359(8.6)$ & $522(13.9)$ & $72(16.9)$ \\
Respiratory complications & $201(4.8)$ & $197(5.2)$ & $25(5.9)$ \\
Cardiac complications & $357(8.5)$ & $372(9.9)$ & $58(13.6)$ \\
Length of stay, median (25th, 75th percentile) & $22.3(14.8-32.9)$ & $28.8(20.2-44.6)$ & $34.8(20.5-49.6)$ \\
In-hospital costs, median (25th, 75th percentile) & $165,272(132,626-203,968)$ & $195,364(153,237-251,809)$ & $253,701(197,116-308,873)$ \\
Discharge disposition (n, \%) & 4014 & 3303 & 224 \\
$\quad$ Home/self-care & $3356(83.6)$ & $2388(72.3)$ & $126(56.3)$ \\
Nursing facility & $566(14.1)$ & $834(25.3)$ & $87(38.8)$ \\
Other & $92(2.3)$ & $78(2.4)$ & $11(4.9)$ \\
\hline
\end{tabular}

$\overline{A K I}$, Acute kidney injury; $A K I-N D$, nondialysis-requiring acute kidney injury; $A K I-D$, acute kidney injury-requiring dialysis; $D V T$, deep vein thrombosis; $P E$, pulmonary embolus.

For example, a study of 86 consecutive patients who underwent continuous-flow LVAD implantation as a bridge to transplantation found that 30 patients $(35 \%)$ required post-LVAD dialysis. ${ }^{7}$ Post-LVAD mortality was $17 \%$ at 1 month, $51 \%$ at 3 months, and $71 \%$ at 6 months; only 6 patients $(20 \%)$ received a heart transplant compared with 39 patients $(70 \%)$ without AKI-D. Larger studies in 3000 to 5000 patients using data from the Interagency Registry for Mechanically Assisted Circulatory Support found that although more than $60 \%$ of patients experienced early improvement in kidney function after LVAD implantation, this improvement is sustained only for a few weeks to months. ${ }^{8,17}$ Patients with preexisting CKD have an especially poor prognosis, particularly if they require dialysis. Post-LVAD mortality for such patients was $30 \%$ at 3 months and $50 \%$ at 3 months for patients requiring dialysis also in cardiogenic shock. ${ }^{8}$ However, these studies did not report outcomes specifically for patients with AKI and did not differentiate between patients with ESRD and AKI-D.

Our study fills this gap by reporting on risk factors for AKI and outcomes among patients who undergo LVAD implantation. The findings are consistent with smaller studies, identifying preexisting CKD, cardiogenic shock, and mechanical ventilation as major risk factors for AKI. ${ }^{7,18-20}$ We suspect the lower risk of AKI associated with valvular heart disease may be related to the exclusion of patients undergoing cardiac surgery procedures at the same time as LVAD implantation from our study, which selected for a patient population with stable or previously fixed valvular heart disease. The lower risk of AKI with hypertension is likely a surrogate for stronger cardiac function or the ability to maintain kidney perfusion. ${ }^{21}$ Despite the trend to implant LVADs in patients who are more hemodynamically stable, ${ }^{3,6}$ our data suggest that there has been little decrease in the rate of AKI (Figure 2 and Table E3). This persistence of AKI may be because LVADs are placed in approximately $40 \%$ of patients with CKD and $30 \%$ of patients requiring mechanical ventilation, factors for AKI that deserve further attention during the LVAD decision-making process.

AKI warrants closer attention for patients considering LVAD implantation because it is one of the strongest risk factors for poor outcomes, which our study confirmed. ${ }^{3,22}$ We observed an association with AKI and common procedural complications such as major bleeding and postoperative sepsis complementing the work of others, ${ }^{7,8}$ with the less common complications approaching the level of statistical significance. The strong association with bleeding and sepsis may be explained by the effects on the coagulation and immune systems that accompany AKI. ${ }^{23-25}$ As expected, we found that patients with AKI$\mathrm{D}$ are a particularly high-risk population, with just more than 1 in 4 patients surviving to be discharged home. These numbers become ever more sobering when accompanied by mechanical ventilation, for which in-hospital mortality rates increase to $70 \%$. Although a small subset of patients with AKI-D do survive for longer periods of time, these high in-hospital mortality rates suggest that LVADs are being offered at a time when patients are actively dying or they may contribute to the dying process.

Our results should help to inform treatment decisions for patients and healthcare providers who are considering LVAD implantation. The literature highlights a need for 


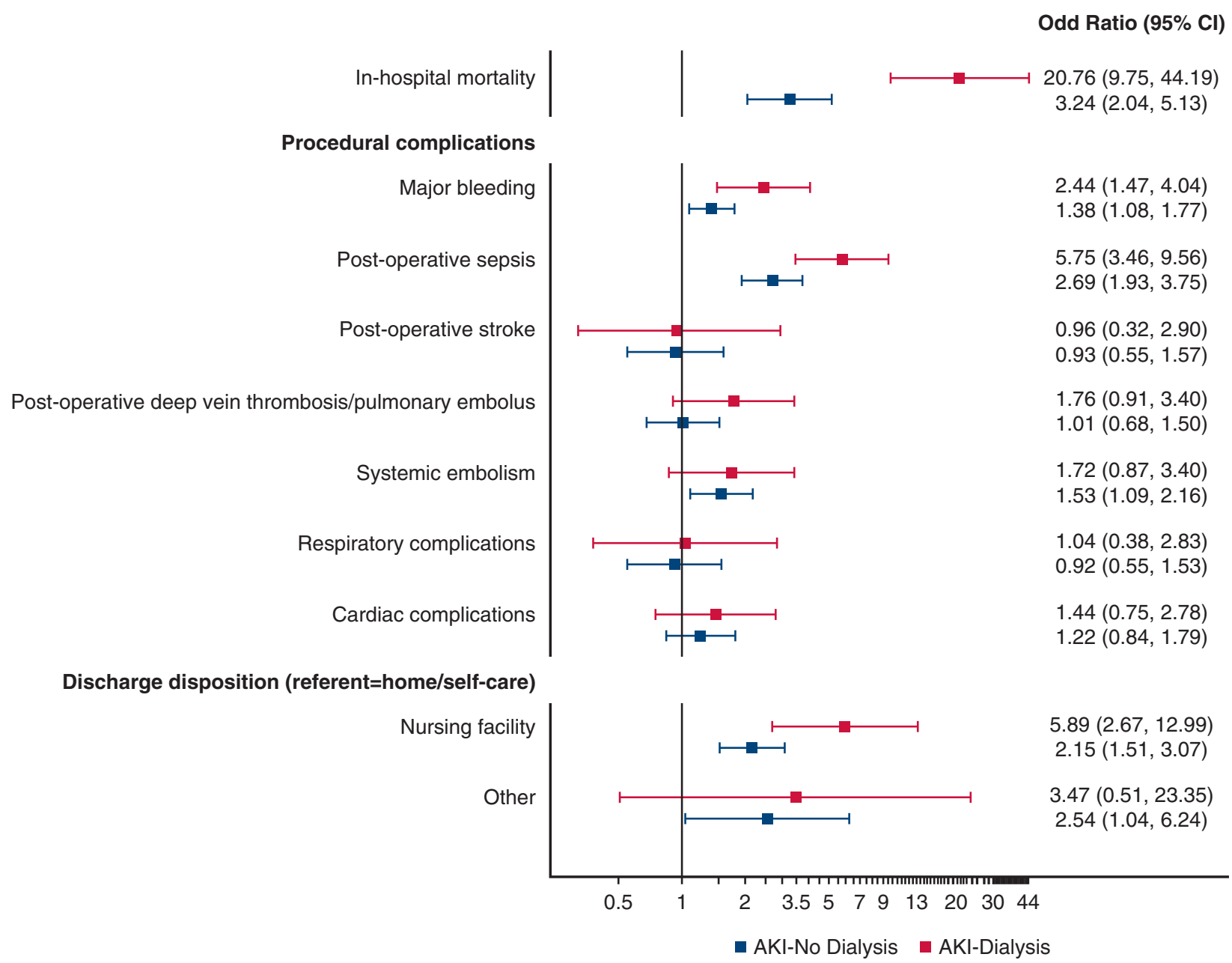

FIGURE 3. Association of AKI with in-hospital mortality, procedural complications, and discharge disposition among patients who underwent implantation of an LVAD. We used logistic regression models to examine the association of AKI-ND and AKI-D versus no AKI (referent group), adjusting for the demographic information, hospital-level variables, patient comorbidities, and hospitalization details in Table 1. Adjusted ORs and 95\% CIs reflecting the magnitudes of these associations are shown. We found that patients with AKI-ND and AKI-D had higher adjusted odds of mortality, major bleeding, postoperative sepsis, and discharge to a nursing facility. AKI, Acute kidney injury; $C I$, confidence interval.

improved shared decision-making to ensure patients appreciate the risks, benefits, and burdens of LVAD implantation, as well as the formulation of realistic expectations postimplantation. ${ }^{26,27}$ Current decision-aids focus overwhelmingly on the benefits, with a recent review demonstrating that $45 \%$ and $63 \%$ of available resources do not mention risks or lifestyle considerations, respectively. ${ }^{28}$ Even when risks are described, AKI and the implications on prognosis are often omitted ${ }^{29}$; this practice is concerning because AKI is a major risk factor for poor outcomes. At 2 years, most patients are satisfied with their decision to pursue LVAD therapy and report improvements in quality of life. ${ }^{3}$ However, patients with AKI-D are likely an exception. Approximately half die in-hospital, and of the survivors, half have substantial self-care needs. These results are consistent with 1 in 4 survivors of AKI-D rating their health-related quality of life equal to or worse than death. ${ }^{30}$
Although these data may not influence patient selection at the time of LVAD implantation when patients often state they have "no choice" given their current health, ${ }^{27,31}$ the poor prognosis in most patients with AKI-D who receive an LVAD should be communicated to patients and caregivers and reinforced after LVAD implantation if patients do not recover sufficient kidney function to stop dialysis.

\section{Study Strengths and Limitations}

Strengths of this study include the use of a nationally representative database, which covers a broad cross section of academic and community hospitals. The NIS possesses data on approximately 8 million hospitalizations annually, so it affords a unique opportunity to examine rare conditions managed in the inpatient setting such as the use of LVADs. Our research team also has experience working with the complex survey design of the NIS and adjusting for patient 
and center case-mix, ${ }^{32-34}$ with the end result that our study is larger and more representative of the US population than previously published studies.

Our study has important limitations. First, we used administrative codes to identify patients with AKI and AKI-D. Although these codes are specific, particularly for AKI-D, they are more likely to identify episodes of moderate to severe AKI compared with mild AKI. ${ }^{14,15}$ We also used administrative codes for some patient comorbidities and hospitalization details, which could lead to misclassification and residual confounding. Second, we excluded patients with evidence of cardiac surgery procedures at the time of LVAD implantation to minimize the well-known association of cardiac surgery with the onset of AKI. Therefore, our findings may underestimate the adverse outcomes associated with AKI. Third, the NIS lacks granularity on the indication for LVAD placement (bridge to transplant or destination therapy) and the type of LVAD placed (pulsatile or continuous flow). We purposely used data from 2008 onward to coincide with the approval of continuous-flow devices, meaning our findings are generally applicable to the implantation of continuous-flow LVADs regardless of indication. Even with the availability of newer devices, there has been little progress in the treatment of AKI over the past decades and technologic advances are unlikely to substantially affect the poor prognosis associated with AKI-D. ${ }^{35}$ Fourth, the NIS lacks some details on the nature and sequence of events during a hospitalization. As a result, we could not determine the timing of an AKI episode (ie, days after hospital admission), its cause, or whether AKI-ND occurred before or after implantation of an LVAD. Further research is needed to determine if outcomes differ on the basis of the onset and cause of AKI, which could have implications for patient selection, management, and counseling before LVAD implantation. Fifth, the NIS does not contain individual patient identifiers, so multiple hospitalizations from the same patient may be represented.

\section{CONCLUSIONS}

Our nationally representative study found that more than 1 in 10 patients with AKI-ND and approximately 1 in 2 patients with AKI-D die during their hospital stay. AKI was associated with higher odds of discharge to a nursing facility, and the outcomes were particularly poor when accompanied by mechanical ventilation. Additional studies are needed to stratify outcomes by the indication for LVAD placement and the timing of AKI onset, as well as provide long-term data on mortality and quality of life that is not available in the NIS. In the meantime, these findings suggest that the presence of AKI and AKI-D should be considered by patients and healthcare providers before and after LVAD implantation, and decision-aids should be updated to reflect both the benefits and the risks associated with LVAD implantation.

\section{Conflict of Interest Statement}

Authors have nothing to disclose with regard to commercial support.

\section{References}

1. Shah N, Agarwal V, Patel N, Deshmukh A, Chothani A, Garg J, et al. National trends in utilization, mortality, complications, and cost of care after left ventricular assist device implantation from 2005 to 2011. Ann Thorac Surg. 2016;101:1477-84

2. Rose EA, Gelijns AC, Moskowitz AJ, Heitjan DF, Stevenson LW, Dembitsky W, et al. Long-term use of a left ventricular assist device for end-stage heart failure N Engl J Med. 2001;345:1435-43.

3. Kirklin JK, Pagani FD, Kormos RL, Stevenson LW, Blume ED, Myers SL, et al Eighth annual INTERMACS report: special focus on framing the impact of adverse events. J Heart Lung Transplant. 2017;36:1080-6.

4. Mulloy DP, Bhamidipati CM, Stone ML, Ailawadi G, Kron IL, Kern JA. Orthotopic heart transplant versus left ventricular assist device: a national comparison of cost and survival. J Thorac Cardiovasc Surg. 2013;145:566-73.

5. Shah N, Chothani A, Agarwal V, Deshmukh A, Patel N, Garg J, et al. Impact of annual hospital volume on outcomes after left ventricular assist device (LVAD) implantation in the contemporary era. J Card Fail. 2016;22:232-7.

6. Patel AM, Adeseun GA, Ahmed I, Mitter N, Rame JE, Rudnick MR. Renal failure in patients with left ventricular assist devices. Clin J Am Soc Nephrol. 2013;8:484-96.

7. Sandner SE, Zimpfer D, Zrunek P, Rajek A, Schima H, Dunkler D, et al. Renal function and outcome after continuous flow left ventricular assist device implantation. Ann Thorac Surg. 2009;87:1072-8.

8. Kirklin JK, Naftel DC, Kormos RL, Pagani FD, Myers SL, Stevenson LW, et al. Quantifying the effect of cardiorenal syndrome on mortality after left ventricular assist device implant. J Heart Lung Transplant. 2013;32:1205-13.

9. Thomas SS, Zern EK, D'Alessandro DA. The renal challenge with left ventricular assist device therapy-when enough is enough. JAMA Intern Med. 2018;178:210-1.

10. Healthcare Cost and Utilization Project (HCUP): HCUP Nationwide Inpatient Sample. Available at: http://www.hcup-us.ahrq.gov/nisoverview.jsp. Accessed January 10, 2016.

11. Kirklin JK, Naftel DC, Pagani FD, Kormos RL, Stevenson LW, Blume ED, et al Sixth INTERMACS annual report: a 10,000-patient database. J Heart Lung Transplant. 2014;33:555-64.

12. Hsu RK, McCulloch CE, Dudley RA, Lo LJ, Hsu CY. Temporal changes in incidence of dialysis-requiring AKI. J Am Soc Nephrol. 2013;24:37-42.

13. Lenihan CR, Montez-Rath ME, Mora Mangano CT, Chertow GM Winkelmayer WC. Trends in acute kidney injury, associated use of dialysis, and mortality after cardiac surgery, 1999 to 2008. Ann Thorac Surg. 2013;95: 20-8.

14. Waikar SS, Wald R, Chertow GM, Curhan GC, Winkelmayer WC, Liangos O, et al. Validity of international classification of diseases, ninth revision, clinical modification codes for acute renal failure. J Am Soc Nephrol. 2006;17:1688-94.

15. Grams ME, Waikar SS, MacMahon B, Whelton S, Ballew SH, Coresh J Performance and limitations of administrative data in the identification of AKI. Clin J Am Soc Nephrol. 2014;9:682-9.

16. Tromp TR, de Jonge N, Joles JA. Left ventricular assist devices: a kidney's perspective. Heart Fail Rev. 2015;20:519-32.

17. Brisco MA, Kimmel SE, Coca SG, Putt ME, Jessup M, Tang WW, et al Prevalence and prognostic importance of changes in renal function after mechanical circulatory support. Circ Heart Fail. 2014;7:68-75.

18. Iwashima Y, Yanase M, Horio T, Seguchi O, Murata Y, Fujita T, et al. Serial changes in renal function as a prognostic indicator in advanced heart failure patients with left ventricular assist system. Ann Thorac Surg. 2012;93:816-23.

19. Hasin T, Topilsky Y, Schirger JA, Li Z, Zhao Y, Boilson BA, et al. Changes in renal function after implantation of continuous-flow left ventricular assist devices. J Am Coll Cardiol. 2012;59:26-36.

20. Alba AC, Rao V, Ivanov J, Ross HJ, Delgado DH. Predictors of acute renal dysfunction after ventricular assist device placement. J Card Fail. 2009;15: 874-81. 
21. Kalantar-Zadeh K, Block G, Horwich T, Fonarow GC. Reverse epidemiology of conventional cardiovascular risk factors in patients with chronic heart failure. J Am Coll Cardiol. 2004;43:1439-44.

22. Genovese EA, Dew MA, Teuteberg JJ, Simon MA, Bhama JK, Bermudez CA, et al. Early adverse events as predictors of 1-year mortality during mechanical circulatory support. J Heart Lung Transplant. 2010;29:981-8.

23. Grams ME, Rabb H. The distant organ effects of acute kidney injury. Kidney Int. 2012:81:942-8

24. Doi K, Rabb H. Impact of acute kidney injury on distant organ function: recent findings and potential therapeutic targets. Kidney Int. 2016;89:555-64.

25. Doi K. Role of kidney injury in sepsis. J Intensive Care. 2016;4:17.

26. Ottenberg AL, Cook KE, Topazian RJ, Mueller LA, Mueller PS, Swetz KM Choices for patients "without a choice": interviews with patients who received a left ventricular assist device as destination therapy. Circ Cardiovasc Qual Outcomes. 2014;7:368-73.

27. Kitko LA, Hupcey JE, Birriel B, Alonso W. Patients' decision making process and expectations of a left ventricular assist device pre and post implantation. Heart Lung. 2016;45:95-9.

28. Iacovetto MC, Matlock DD, McIlvennan CK, Thompson JS, Bradley W, LaRue SJ, et al. Educational resources for patients considering a left ventricular assist device: a cross-sectional review of internet, print, and multimedia materials. Circ Cardiovasc Qual Outcomes. 2014;7:905-11.

29. Thompson JS, Matlock DD, McIlvennan CK, Jenkins AR, Allen LA Development of a decision aid for patients with advanced heart failure considering a destination therapy left ventricular assist device. JACC Heart Fail. 2015:3:965-76.

30. Johansen KL, Smith MW, Unruh ML, Siroka AM, O'Connor TZ, Palevsky PM, et al. Predictors of health utility among 60-day survivors of acute kidney injury in the veterans affairs/National Institutes of Health acute renal failure trial network study. Clin J Am Soc Nephrol. 2010:5:1366-72.

31. Mcllvennan CK, Allen LA, Nowels C, Brieke A, Cleveland JC, Matlock DD Decision making for destination therapy left ventricular assist devices "there was no choice" versus "I thought about it an awful lot" Circ Cardiovas Qual Outcomes. 2014;7:374-80.

32. Silver SA, Long J, Zheng Y, Chertow GM. Cost of acute kidney injury in hospitalized patients. J Hosp Med. 2017;12:70-6.

33. Chong K, Silver SA, Long J, Zheng Y, Pankratz VS, Unruh ML, et al. Infrequent provision of palliative care to patients with dialysis-requiring AKI. Clin J Am Soc Nephrol. 2017;12:1744-52.

34. Bradshaw C, Zheng Y, Silver SA, Chertow GM, Long J, Anand S. Acute kidney injury due to diarrheal illness requiring hospitalization: data from the Nationa Inpatient Sample. J Gen Intern Med. 2018;33:1520-7.

35. Siew ED, Himmelfarb J. The inexorable rise of AKI: can we bend the growth curve? J Am Soc Nephrol. 2013;24:3-5.

Key Words: acute kidney injury, heart failure, left ventricular assist device 
TABLE E1. ICD-9 codes used to define study criteria, comorbidities, and procedures

\section{Disease or procedure}

ICD-9 code*

Inclusion criteria
Implant of LVAD

Exclusion criteria

End-stage renal disease

Biventricular assist device

Short-term mechanical circulatory support placement

Cardiac surgery procedures

Lung transplant

Combined heart-lung transplant

Open heart valvuloplasty without replacement

Heart valve replacement

Operating on structures adjacent to heart valves

Production of septal defect in heart

Repair of congenital cardiac anomalies

Other operations on valves and septa of heart

Transmyocardial revascularization

Other operations on vessels of heart

Cardiotomy and pericardiotomy

Pericardiectomy and excision of heart lesion

Repair of heart and pericardium

Replacement/repair of total replacement heart system

Removal of internal biventricular heart system

Implantation of heart and circulatory assist system

Resection of aortic/thoracic vessel with anastomosis

Resection of aortic/thoracic vessel with replacement

Endovascular implantation of graft in thoracic aorta/other vessels

Exposure

Acute kidney injury

AKI-D

Covariates and comorbidities

Atrial fibrillation

Cancer

CKD

Chronic obstructive pulmonary disease

Congestive heart failure

Coronary artery disease

Dementia

Diabetes

History of coronary artery bypass grafting

History of percutaneous coronary intervention

Hypertension

Liver disease

Peripheral vascular disease

Valvular heart disease
Procedure code 37.66

Diagnosis code 585.6

Procedure code $39.27,39.42,39.43,39.93,54.98$ or One of the following codes without diagnosis code 584 :

Diagnosis code V45.1, V56.0, V56.1, V56.2, V56.3, V56.8

Procedure code 39.95

Procedure code 37.52

Procedure code 37.60, 37.62, 37.65, 37.68

Procedure code

33.5

33.6

35.1

35.2 (except $35.22 \rightarrow$ other replacement of mitral valve)

35.3

35.4

35.8

35.9 (except $35.97 \rightarrow$ percutaneous mitral valve repair)

36.3

36.9

37.1

37.3 (except $37.34,37.37 \rightarrow$ endovascular/thoracoscopic destruction of heart lesion)

37.4

$37.53,37.54$

37.55

37.6 (except $37.61 \rightarrow$ intra-aortic balloon pump and $37.66 \rightarrow$ LVAD)

$38.34,38.35$

$38.44,38.45$

$39.73,39.79$

Diagnosis code 584

584 plus one of the following:

Diagnosis code V45.1, V56.0, V56.1

Procedure code 39.95

Diagnosis Code

427.3

140-209, 238.4-238.7

$250.4,403,404,585.1,585.2,585.3,585.4,585.5,585.9,586$

$491,492,496$

$425,428,514,518.4$

$410,411,412,413,414,429.7$

$290,294.1,294.2,331.0,331.1,331.2,331.82,797$

250

Diagnosis code V45.81

Procedure code 36.1

Procedure code $00.66,36.06,36.07$

401-405, 437.2

$456.1,070,571,572.2,572.3,572.4,572.8,573,782.4,789.1,789.5$, V02.6

$440,443.1,443.81,443.82,443.89,443.9, \mathrm{~V} 43.4$

$394,395,396,397,424.0,424.1,424.2,424.3$ 
TABLE E1. Continued

Disease or procedure

Covariates, details from acute hospitalization

Acute respiratory distress syndrome

Cardiogenic shock

Cardioplegia

Closure of septal defects

Intra-aortic balloon pump

Mechanical ventilation

Swan-Ganz insertion
ICD-9 code

Procedure code

96.7 plus 1 of the following:

Diagnosis code $518.5,518.81,518.82$

Diagnosis code 785.51

39.63

$35.5,35.6,35.7$

37.61

$96.04,96.05,96.7$

$89.63,89.64,89.66,89.67,89.68$

ICD-9, International Classification of Diseases, 9th Revision; $L V A D$, left ventricular assist device; $A K I-D$, acute kidney injury-requiring dialysis; $C K D$, chronic kidney disease *If a higher level code is listed, all lower-level codes are also included. For example, if ICD-9 code 584 is listed, then all of 584.5, 584.6, 584.7, 584.8, 584.9 were also included. 
TABLE E2. ICD-9 codes used to define secondary outcomes

\section{Procedural complication}

Major bleeding

- postoperative hemorrhage or hematoma

- intracranial bleed

- hemoperitoneum

- gastrointestinal bleed

- genitourinary bleed

- hemoptysis/epistaxis

Postoperative sepsis (Patient Safety Indicator definition)

Postoperative stroke

Postoperative DVT or PE (Patient Safety Indicator definition)

Systemic embolism

Respiratory complications

- pneumothorax or other iatrogenic respiratory complication

Cardiac complications

- complete heart block, pericardial complication, or other iatrogenic cardiac complication
ICD-9 diagnosis code*

430, 431, 432.0, 432.1, 432.9, 456.0, 456.2, 459.0, 530.21, 530.7, $530.82,531.0,531.2,531.4,531.6,532.0,532.2,532.4,532.6,533.0$, $533.2,533.4,533.6,534.0,534.2,534.40,534.6,535.0,535.21$, $535.31,535.41,535.51,535.61,535.71,537.83,537.84,562.02$, 562.03, 562.12, 562.13, 568.81, 569.3, 569.85, 569.86, 578.0, 578.1, $578.9,596.7,599.70,599.71,784.7,786.3,998.11,998.12$

038.0, 038.1, 038.2, 038.3, 038.40, 038.41, 038.42, 038.43, 038.44, $038.49,038.8,038.9,785.52,785.59,995.91,995.92,998.00,998.02$ 435.0, 435.1, 435.2, 435.3, 435.8, 435.9, 997.02, V12.54

$415.1,415.11,415.13,415.19,451.11,451.19,451.2,451.81,451.9$, $453.40,453.41,453.42,453.8,453.9$

$289.59,362.30,362.31,362.32,362.33,362.34,433,434,444.0,444.1$, $444.2,442.8,442.9,557.0,573.4,593.81,996.70,996.72$

$512.1,997.31,997.39$

Diagnosis code 997.1, 423.0, 423.3, 426.0

Procedure code $37.71,37.72,37.78,37.80,37.8137 .82,37.83,39.64$

$\overline{I C D-9, \text { International Classification of Diseases, 9th Revision; } D V T \text {, deep vein thrombosis; } P E \text {, pulmonary embolus. *If a higher level code is listed, all lower-level codes are also }}$ included. For example, if ICD-9 code 584 is listed, then all of 584.5, 584.6, 584.7, 584.8, and 584.9 were also included.

TABLE E3. Use of left ventricular assist devices stratified by year

\begin{tabular}{|c|c|c|c|c|c|c|c|c|c|}
\hline \multirow[b]{2}{*}{ Year } & \multicolumn{3}{|c|}{ No AKI $(n, \%)$} & \multicolumn{3}{|c|}{ AKI-ND (n, \%) } & \multicolumn{3}{|c|}{ AKI-D (n, \%) } \\
\hline & Unadjusted & Model 1* & Model 2† & Unadjusted & Model 1* & $\overline{\text { Model } 2 \dagger}$ & Unadjusted & Model 1* & Model 2 $†$ \\
\hline 2008 & 404 (51.4) & $402(51.1)$ & $393(50)$ & $342(43.5)$ & $345(43.9)$ & $356(45.3)$ & $40(5.1)$ & $39(5.0)$ & $36(4.7)$ \\
\hline 2009 & $583(52.1)$ & $586(52.4)$ & $608(54.4)$ & $480(42.9)$ & 477 (42.7) & $460(41.1)$ & $55(5.0)$ & $54(4.9)$ & $50(4.5)$ \\
\hline 2010 & $795(55.1)$ & 793 (54.9) & $781(54.1)$ & $562(39.0)$ & $565(39.2)$ & $579(40.2)$ & $85(5.9)$ & $84(5.9)$ & $82(5.7)$ \\
\hline 2011 & $701(45.1)$ & $699(45.0)$ & $729(46.9)$ & $781(50.3)$ & $783(50.4)$ & $756(48.7)$ & $71(4.6)$ & $71(4.6)$ & $68(4.4)$ \\
\hline 2012 & $860(51.6)$ & $860(51.6)$ & $830(49.9)$ & 710 (42.6) & 709 (42.6) & $733(44.0)$ & $95(5.8)$ & $96(5.8)$ & $102(6.1)$ \\
\hline 2013 & $925(46.8)$ & $926(46.9)$ & $923(46.7)$ & 960 (48.6) & $958(48.5)$ & $953(48.3)$ & $90(4.6)$ & $90(4.6)$ & $99(5.0)$ \\
\hline
\end{tabular}

$A K I$, Acute kidney injury; $A K I-N D$, nondialysis-requiring AKI; AKI-D, AKI-requiring dialysis. *Model 1 is adjusted for age and sex. $\dagger$ Model 2 is adjusted for age, sex, admission type, CKD, hypertension, valvular heart disease, cardiogenic shock, and mechanical ventilation. 